\title{
Buchbesprechungen
}

Hans-Jürgen Rösner und Frank SchulzNieswandt (Hrsg.), Beiträge der genossenschaftlichen Selbsthilfe zur wirtschaftlichen und sozialen Entwicklung - Bericht der XVI. Internationalen Genossenschaftswissenschaftlichen Tagung 2008 in Köln, Neue Kölner Genossenschaftswissenschaft, Band 5, Lit Verlag, Berlin 2009, $728 \mathrm{~S}$.

Alle vier Jahre führt die Arbeitsgemeinschaft Genossenschaftswissenschaftlicher Institute (AGI) ihre Internationale Genossenschaftswissenschaftliche Tagung (IGT) durch. Das Ergebnis der jüngsten Tagung haben die beiden Herausgeber durch eine aktuelle und umfangreiche Zusammenstellung der wissenschaftlichen Beiträge zum Genossenschafts- und Kooperationswesen in den beiden vorliegenden Tagungsbänden dokumentiert.

Das Themengebiet des Genossenschaftswesens zeichnet sich durch eine große Vielfalt der mit Kooperationsfragen befassten wissenschaftlichen Fachdisziplinen und einem breiten praktischen Anwendungsspektrum aus. Naturgemäß kommen demnach auf Fachkongressen eine große Anzahl an Beiträgen und eine Vielzahl an unterschiedlichen Perspektiven auf das Fachgebiet zusammen. Der aus zwei Bänden bestehende Tagungsbericht stellt ein dementsprechendes Abbild der aktuellen internationalen genossenschaftswissenschaftlichen als auch -praktischen Diskussion dar. Die einzelnen Diskussionsbeiträge spiegeln die wirtschaftlichen und sozialen Herausforderungen für Genossenschaften deutlich wider und zeigen auf, auf welchem theoretischen Fundament die Genossenschafts- und Kooperationsforschung sich bewegt. Gezeigt werden die unterschiedlichen Antworten auf die besonderen Entwicklungen der Genossenschaften insbesondere im internationalen Kontext. Besondere Berücksichtigung findet in den beiden Bänden die Rolle der Genossenschaften als Unternehmen und ihre Rolle im Zusammenspiel mit institutionellen Veränderungen im Rahmen der sozialen und wirtschaftlichen Entwicklung vor dem Hintergrund ihres besonderen rechtlichen und wirtschaftlichen Status.
Gemäß des Generalthemas der internationalen Tagung „Genossenschaftliche Selbsthilfe zur wirtschaftlichen und sozialen Entwicklung" werden die Beiträge drei thematischen Schwerpunkten zugeordnet. So werden die genossenschaftlichen Bezüge im Rahmen der Mittelstandsentwicklung, der sozialen Dienstleistungen und der Entwicklungsförderung hergestellt und aus sozial-ökonomischer und rechtlicher Sicht gewürdigt. Diese Schwerpunktsetzung durchzieht die beiden Bände wie ein roter Faden. Eingefasst werden die Schwerpunktthemen durch umfangreichere Beiträge zur wissenschaftlichen Grundlegung und zu möglichen zukünftigen Forschungsinhalten.

Abgesehen von der Vielfalt der in den Tagungsbänden unterbreiteten theoretischen und praktisch-orientierten Themen, gewinnt das vorliegende Werk dadurch einen besonderen Charme, dass an prominenter Stelle zwei Nobelpreisträger ausführlich Gehör finden und durch ihre Grundsatzbeiträge den Sammelband bereichern. Im Rahmen der Tagung wurde dem Friedensnobelpreisträger von 2006, Prof. Muhammad Yunus, der Internationale Wissenschaftspreis der AGI verliehen. Seine hier abgedruckte Ansprache, die er im Rahmen der Preisverleihung hielt, zeigt nochmals eindrucksvoll auf, wie die Genossenschaftsidee in ihrer grundsätzlichen Form hilfreich bei der Entwicklung von praktischen Organisationsformen zur Linderung der existenziellen Not in Entwicklungsländern erfolgreich beitragen kann. Besonders lesenswert ist auch der Beitrag der Wirtschaftsnobelpreisträgerin von 2009, Prof. Elinor Ostrom, die in ihrem Vortrag aufzeigt, wie gemeinschaftliches Eigentum effektvoll genossenschaftlich organisiert werden kann.

In seinem Hauptreferat skizziert Wolfgang Hardtwig (Berlin) die wesentlichsten Merkmale genossenschaftlicher Personenverbindungen, die aus seiner Sicht seit über 800 Jahren den Gesellschaftsaufbau und die und seine kulturellen Ausformungen sozial- und wirtschaftsprinzipiell geprägt haben. In seinem, nicht nur für den geschichtlich bewanderten Leser, interessanten Beitrag vermittelt der Au- 
tor die Grundprinzipien der deutschen Genossenschaftsentwicklung in seinen wesentlichen Ordnungsmustern und Organisationsausformungen über die Jahrhunderte hinweg. Zwei weitere Grundsatzpapiere eröffnen die Internationale Tagung und geben den thematischen Rahmen für die folgenden Schwerpunktthemen vor. So weist Elinor Ostrom (Bloomington, USA) mit ihrem Beitrag auf den Zusammenhang von genossenschaftlichen Organisationsformen und der Theory of Collective Action hin. Dabei stellt sie besonders die Bedeutung menschlicher Verhaltenskomponenten bei Entscheidungen, wie etwa die begrenzte Rationalität oder auch das durch Normen geprägte Entscheidungsverhalten als die treibenden Kräfte für die Bildung und Festigung sozialer Kooperationen heraus. Ihrer Ansicht nach sind Phänomene wie etwa Reziprozität oder Reputation, die Individuen bei ihren Entscheidungen berücksichtigen, in Kooperation wichtige Voraussetzungen für die Beseitigung sozialer Dilemmata. Zu den bisher noch nicht erforschten komplexen Zusammenhängen zwischen den Einflussfaktoren und der empirischen Überprüfung gibt sie einige interessante Empfehlungen für die weitere wissenschaftliche Auseinandersetzung. Einige dieser Grundgedanken werden in dem Beitrag von Axel Ockenfels und Felix Lamouroux (Köln) theoretisch untermauert und an Hand eines praktischen Beispiels aus dem Internethandel vertieft. In ihrem Paper zeigen sie deutlich auf, welche Reputationsmechanismen durch ein institutionelles Design eingeführt werden müssten, um ein fundamentales Vertrauensdilemma in Kooperationsbeziehungen zu überwinden. Durch die Wahl und bewusste Gestaltung verschiedener Kooperationssysteme ist man in der Lage, so die beiden Autoren, die Gefahr des negativen reziproken Verhaltens der Kooperationsteilnehmer zu reduzieren und in Kooperationsbeziehungen die Basis für eine vertrauensvolle Zusammenarbeit zu legen.

Im Rahmen der wissenschaftlichen Grundlegung folgen im ersten der beiden Bände dann jeweils drei beziehungsweise vier Einzelbeiträge zu den Schwerpunktthemen Genossenschaften im Umfeld der Mittelstandsentwicklung, der sozialen Dienstleistungen und der Entwicklungsförderung. Man mag sicherlich über die eine oder andere Beitragszuordnung zu einem dieser Generalthemen unterschiedli- cher Auffassung sein, generell gilt allerdings, dass in den insgesamt 11 Beiträgen eine Fülle genossenschaftsrelevanter aktueller Themen aus unterschiedlichen Fachdisziplinen beleuchtet werden. So werden genossenschaftsinstitutionelle Aspekte angesprochen, indem zum Beispiel Jörg Sydow (Berlin) zur Vorsicht bei der Übertragung des Netzwerkgedankens auf Genossenschaften mahnt oder Annette Zimmer (Münster) der Frage nachgeht, inwiefern Genossenschaften als zivilgesellschaftliche Organisationen angesehen werden können. Ein interessanter Themenkomplex ergibt sich aus den Fragen ob und in welchem Umfang Genossenschaften geeignete Kooperationsformen darstellen, um im kommunalen und öffentlichen Bereich Leistungen zur allgemeinen Daseinsvorsorge anzubieten (Gabriel Obermann, Wien) oder welche Rolle eine genossenschaftlich organisierte Wohlfahrtsproduktion spielen kann (Beate Finis-Siegler, Frankfurt am Main). Daneben erfahren genossenschaftsrechtliche Fragen eine Grundlegung, wie etwa Fragen zur Gründungserfahrung bei der europäischen Genossenschaftsrechtsform (Mathias Fiedler, Hamburg). Hagen Henrÿ von der ILO aus Genf geht der Frage nach, inwieweit der genossenschaftliche Rechtsrahmen und die genossenschaftliche Unternehmensformen generell die besseren Voraussetzungen für eine nachhaltige Entwicklung im Vergleich $\mathrm{zu}$ anderen Organisationsformen bieten können. Weitere Beiträge nehmen zu genossenschaftlichen Managementproblemen Stellung, indem die Führung genossenschaftlicher Verbundsysteme thematisiert werden (Johannes Blome-Drees, Köln), die Frage nach Genossenschaft als Marke untersucht wird (Andreas Aulinger, Berlin) oder die Rolle genossenschaftlicher Produzentenorganisationen und ihre vertikale Koordinationsfunktion grundsätzlich beleuchtet wird (Jos Bijman und Meike Wollni, Wageningen).

Der zweite Band beleuchtet Probleme der Genossenschaftspraxis aus Sicht der Wissenschaft, der Genossenschaftsverbände und den Genossenschaften selbst. Eröffnet wird dieser thematische Schwerpunkt durch einen Beitrag von George Hendrikse u. a. (Rotterdam) mit dem gleichwohl interessanten wie gelungenen Versuch, Genossenschaften - hier Verarbeitungsgenossenschaften - unter verschiedenen Organisations- und Governancemustern (Sys- 
tem of Attributes) gegenüber nicht-genossenschaftlichen Unternehmensformen abzugrenzen. Eine Vielzahl von Beiträgen widmet sich dem Thema Genossenschaften und soziale Dienstleistungen und der Rolle genossenschaftlicher Organisationsformen in der kommunalen Versorgung. In diesen Beiträgen wird neben der Versorgung mit Wohnungen als wesentlichem Förderungsauftrag von Wohnungsgenossenschaften auch das Angebot an zusätzlichen Maßnahmen des Sozialmanagements für die Mitglieder angesehen (Iris Beuerle, Hamburg), wie zum Beispiel die private Altersvorsorge durch Wohnbeteiligungsangebote mit weiteren Geschäftsanteilen zu unterstützen (Monika Kegel, Berlin) oder auch mehr praktische Umsetzungsalternativen, wie etwa eine Beschreibung neuer Wohnmodelle des Mehrgenerationswohnens auf genossenschaftlicher Basis (Georg Potschka, Köln) oder die genossenschaftlichen Antworten auf die Anforderungen eines Angebots an altersgerechtem Wohnen (Stefan Keim, Gera). Ebenso werden in diesen, mehr sich an praktischen Problemen orientierenden Beiträgen auch alternative Finanzierungsinstrumente für Wohnungsgenossenschaften (Hubert Scharlau, Lünen) und das gesunde Wohnen als ein Aktionsfeld für Genossenschaften vorgestellt (Elke Schlaginweit und Kerstin Schulte-Eckel, Nürnberg). Insgesamt zeigen die Beiträge in diesem Themenfeld die vielfältigen Aufgaben, die genossenschaftlichen Wohnungs- und Sozialeinrichtungen zugesprochen werden und die die Unternehmensrechtsform in der Lage ist zu leisten. Sowohl aus mehr wissenschaftlich-theoretischer Sicht als auch aus praktischer Erfahrungsperspektive liefern die Beiträge einen interessanten Einblick in den sozialen Charakter, den Genossenschaften durch die Übernahme von sozialen Dienstleistungen bieten können.

An diese Perspektiven schließen sich eine Reihe weiterer Beiträge an, die sich vornehmlich mit der Frage nach der Eignung genossenschaftlicher Organisationsformen und-modelle für die Übernahme kommunaler Versorgungsleistungen beziehen. So wenden sich Dietmar Rössl und Isabella Hatak (Wien) der wichtigen Frage zu, ob Genossenschaften als Modelle für neue zwischenbetriebliche Kooperationen, im Sinne sogenannter Public-Citizen-Partnerships, angesehen werden können.
In weiteren Beiträgen wird die Rolle der Genossenschaften als Modell für kooperative Formen von Wohlfahrtsverbänden untersucht, mit dem Ziel, deren wirtschaftliche Zwänge zu mildern und dem Zweck, aus der Sicht der Sozialgenossenschaftlichkeit, die Zufriedenheit der Mitglieder, die Kooperationsbereitschaft und letztendlich die Produktivität zu verbessern (Peter-Georg Albrecht, Magdeburg). Zwei Beiträge zu institutionellen Genossenschaftskonzepten, das genossenschaftliche Franchisesystem für haushaltsnahe Dienstleistungen (Burghard Flieger, Freiburg) und zu einer genossenschaftsverträglichen Individualisierung von Rücklagen (Holger Blisse, Wien) runden das vielfältige Bild zu Genossenschaften $\mathrm{ab}$.

Im dritten Schwerpunkt der Veranstaltung werden aus unterschiedlichen Fachdisziplinen Zukunftsperspektiven genossenschaftsbezogener Forschung dargelegt. Dabei werden zahlreiche Fragen aufgeworfen, so etwa die Frage danach, welche Auswirkungen Traditionszusammenhänge und unterschiedliche Leitungs- und Entscheidungsstrukturen, die sich bei den vielfältigen Formen genossenschaftlich-kooperativer Selbsthilfe, des bürgerschaftlichen Engagements und auch der Tätigkeit von Stiftungen zeigen, auf die Zukunft der Bürgergesellschaft haben (Wilhelm Krull, Hannover). Ebenso wird aus Sicht der Institutionellen Ressourcenökonomik der Frage nachgegangen, ob Genossenschaften, im betrachteten Fall Agrargenossenschaften, als integrative oder eher als segregative Institutionen angesehen werden sollten. Deutlich wird aufgezeigt, dass zu einer befriedigenden Beantwortung der Frage ein weiterer empirischer Forschungsbedarf benötigt wird (Konrad Hagedorn, Berlin). In einem weiteren Beitrag geht es insbesondere um die Möglichkeiten der $\mathrm{Zu}$ kunftssicherung der genossenschaftlichen Rechtsform. Anhand der Beobachtung, dass in der betriebswirtschaftlichen Literatur zu Kooperation und Vernetzung kaum von genossenschaftlichen Aspekten die Rede ist, werden einzelne Modelle neuer Genossenschaftstypen erarbeitet, die das Problem der Unterrepräsentanz des Genossenschaftsmodells in der Betriebswirtschaftslehre beheben und $\mathrm{zu}$ mehr Akzeptanz führen können (Harald Bolsinger, Nürnberg). Mit Sektor- und länderspezifischen Ausführungen zu wichtigen Forschungsansät- 
zen zur organisatorischen Restrukturierung von landwirtschaftlichen Genossenschaften (Constantine Iliopoulos, Athen) und zu einem neuen Partizipationsmodell für schwedische Dienstleistungsgenossenschaften (Victor Pestoff, Östersund) wird der als Forschungsdesiderate bezeichnete thematische Schwerpunkt abgeschlossen.

Auf insgesamt 680 Seiten findet sich die aktuelle und gesamte Bandbreite genossenschaftsspezifischer Diskussionsbeiträge eines internationalen Forschungskreises, der auf Einladung der Arbeitsgemeinschaft genossenschaftswissenschaftlicher Institute (AGI) zur Internationalen Tagung (IGT) seine wissenschaftlichen Forschungsergebnisse und Diskussionsbeiträge präsentiert hat. Insgesamt 41 wissenschaftliche und teilweise praxisorientierte Beiträge finden sich in den beiden Bänden wieder. Dabei ist nicht mitgerechnet, dass in beiden Bänden auch die Gesprächsabläufe zweier Podiumsdiskussionen wiedergegeben werden. Abgeschlossen wird der Tagungsband durch ein Autorenverzeichnis, sowie einen kurzen Abriss über die Geschichte der IGT.

Den beiden Herausgebern ist zu danken, dass sie sich der Mühe unterzogen haben, mit den beiden Tagungsbänden ein umfassendes und die Bandbreite der Genossenschaftswissenschaft abdeckende Schrift vorzulegen. Das Werk vermittelt dem interessierten Forscher und Praktiker einen guten Überblick über den Stand und die Weiterentwicklungen der Genossenschafts- und Kooperationsforschung. Insbesondere sollte sich der Leserkreis der Zö$\mathrm{gU}$ durchaus von dieser Veröffentlichung angesprochen fühlen, da nicht nur die genossenschaftliche Unternehmensform berücksichtigt wird. In zahlreichen Beiträgen wird eindrucksvoll gezeigt, welche Möglichkeiten genossenschaftliche Organisations- und Führungsmodelle als Dienstleistungsinstitutionen und kommunale Versorgungseinrichtungen für den öffentlichen und gemeinnützigen Sektor bieten können. Der vorliegende Tagungsband kann insgesamt als ein gelungenes Werk eingeschätzt werden, das die Bandbreite der genossenschaftswissenschaftlichen Diskussion widerspiegelt. Die systematische Zusammenstellung der Einzelbeiträge überzeugt. Insgesamt handelt es sich um ein empfehlenswertes Werk, das durch seine integrative, theorie- und praxisorientierte Aufarbeitung der unter- schiedlichen Facetten des Genossenschaftsund Kooperationswesens besticht.

\section{Rainer Kühl}

Edoardo Ongaro, Public Management Reform and Modernization - Trajectories of Administrative Change in Italy, France, Greece, Portugal and Spain, Edward Elgar, Cheltenham (UK)-Northampton, MA (US) 2009, $316 \mathrm{~S}$.

In den vergangenen zwei Jahrzehnten hat es in zahlreichen Staaten umfangreiche Managementreformen im öffentlichen Sektor gegeben, die teilweise unter dem Label „New Public Management"stattfanden. Über die diesen Reformen zugrunde liegenden Konzepte - weniger über empirische Resultate - hat es umfangreiche Publikationen gegeben, die meisten davon in englischer Sprache. Der größte Teil der vorhandenen Literatur bezieht sich allerdings auf Managementreformen in Nord- und Westeuropa. Südeuropa ist in dieser Hinsicht bislang weitgehend ein blinder Fleck geblieben, was zumindest teilweise an sprachlichen $\mathrm{Zu}$ gangsbarrieren gelegen haben mag. Edoardo Ongaro, der Public Management an der Bocconi Universität in Mailand lehrt, ist angetreten, diese Lücke zu schließen: Er befasst sich im vorliegenden Buch mit Public Management Reformen in fünf südeuropäischen Staaten, die oft gerne unter der Rubrik „Napoleonische Staatstradition" zusammengefasst werden. Der Autor hat sich dabei zum Ziel gesetzt, die verschiedenen Reformthemen und ihre Umsetzung im Zeitverlauf zu beschreiben und vergleichend zu analysieren. Dies tut er in einer umfassenden Sichtweise, die über enge managerielle Konzept- und Instrumentenanalysen weit hinausgeht und auch den politisch-administrativen Kontext der Verwaltungssysteme in den verschiedenen Ländern einbezieht.

Das Buch besteht aus zwei ziemlich ungleichen Teilen: Im ersten Teil führt der Autor eine intensive und detaillierte Analyse der Reformlandschaft in seinem Heimatland Italien durch. Im zweiten Teil schließen sich sehr kompakte, überblicksartige Darstellungen wichtiger Reforminitiativen aus den übrigen vier Ländern an (Frankreich, Griechenland, Portugal und Spanien). Ferner präsentiert Ongaro hier auch eine ausführliche vergleichende Analyse der Reformen in den betrachteten Ländern und 
fügt schließlich eine Untersuchung von Entwicklungsperspektiven in diesen Ländern an. Empirisch stützt sich das Buch einerseits auf die Befunde eines internationalen Forschungsnetzwerks, dem auch der Autor angehört und das sich mit Organisations- und Managementthemen - vor allem in Bezug auf verselbständigte öffentliche Einrichtungen - befasst. Zum anderen hat Ongaro ein umfangreiches Netzwerk südeuropäischer Verwaltungsforscher nutzen können, aus dem er all die Informationen beziehen konnte, die er zur vergleichenden Fünf-Länder-Studie benötigte. Darüber hinaus hat er als Mitglied der führenden Management School Italiens sowie als erfahrener Verwaltungsberater und -forscher natürlich auf umfassende Quellen zu den Reformprozessen und -ergebnissen in Italien zurückgreifen können. In der umfangreichen Einleitung führt der $\mathrm{Au}$ tor in die Problemstellung und Forschungsfragen ein und erläutert sein methodisches Vorgehen. Im umfangreichen Teil I zu Italien, der aus 4 Kapiteln besteht, werden zunächst das politische System des Landes und die Rolle von Parlament und Exekutive sowie die bestehenden Konflikte anschaulich dargestellt. Es folgt eine ausführliche Beschreibung und Kommentierung der wichtigen Managementreformen seit Beginn der 90er Jahre. Dabei werden Reformen im Finanzmanagement, in der Rechnungsprüfung, im Performance Measurement, im Personalbereich sowie in der Verwaltungsorganisation getrennt betrachtet. Das letztgenannte Thema wird sehr ausführlich behandelt, es stehen sowohl Makroreformen (Föderalisierung, Dezentralisierung, Koordination) wie auch Mikroreformen auf Ebene der einzelnen öffentlichen Einrichtungen zur Diskussion. Teil I wird abgeschlossen durch eine umfangreiche theoriegesteuerte Analyse und Erklärung der Verlaufsformen und Ergebnisse von Managementreformen in Italien.

In Teil II präsentiert Edoardo Ongaro nunmehr kurze Profile der Verwaltungssysteme in Frankreich, Griechenland, Portugal und Spanien sowie der in den letzten zwei Jahrzehnten dort durchgeführten Reformen. Dem folgt eine vergleichende Analyse der Reformdynamiken in allen fünf Ländern (also unter Einschluss Italiens), die sich auf neun Kriterien stützt, unter denen Merkmale des öffentlichen Dienstes eine besondere Rolle spielen (z. B. die Rolle von Corps, nicht allein in Frankreich, sondern auch in den anderen Staaten). Im Anschluss wird der Untersuchungsfokus erweitert, indem auch der Einfluss des politisch-administrativen Kontexts des jeweiligen Verwaltungssystems auf die Managementreformen beleuchtet wird. Beispielsweise wird untersucht, wie stark der jeweilige öffentliche Sektor mit NPM durchdrungen worden ist, inwiefern es Implementationslücken bzw. -verzögerungen gegeben hat, welche Rolle die Europäisierung bei den Reformen gespielt hat und inwieweit sich die jeweilige Dezentralisierungspolitik des Landes auf die Reformen ausgewirkt hat. In diesem Abschnitt zeigt der Autor vornehmlich offene Forschungsfragen auf und macht Vorschläge zur künftigen Strukturierung der Forschungslandschaft.

Im letzten Kapitel werden die Staats- und Verwaltungssysteme der behandelten südeuropäischen Länder breiter ausgeleuchtet. Beispielsweise untersucht der Autor die Rolle herrschender globaler Paradigmen in den Ländern. Ein besonderes Augenmerk gilt dabei dem Leitbild des „neo-Weberianischen Staates“, das vor einigen Jahren von Christopher Pollitt und Geert Bouckaert als mögliches NachfolgeModell von NPM in die internationale Debatte eingeführt worden ist. Ongaro erörtert hier geistreich die fünf südeuropäischen Verwaltungssysteme, die teilweise weder, ,richtig Weberianisch" waren noch im Kern infolge der NPM-Welle ,managerialisiert" worden sind. Immer wieder wird hier deutlich, wie vielgestaltig doch die Verwaltungskulturen und -strukturen in Europa sind und wie wenig Sinn es macht, sie in den Reformdebatten ,über einen Kamm zu scheren".

Das hier vorgestellte Buch von Edoardo Ongaro ist ein wertvoller „Lückenfüller“: Es bereichert die internationale Debatte zu Public Management-Reformen um die bisher oftmals ausgeblendete südeuropäische Perspektive. Es macht dem Leser deutlich, dass viele Reformen im Süden ganz anders als im Rest von Europa gelaufen sind und dass dort auch abweichende Reformthemen eine Rolle gespielt haben. Darüber hinaus erklärt der Autor auf Basis aktueller Theoriekonzepte die Unterschiede in den einzelnen Ländern, aber auch die Besonderheiten dieser napoleonischen Staaten im Vergleich zu anderen Staatstraditionen in Europa. Vor allem zu Italien erfährt man aus dem Buch spannende Details und Trends, was angesichts 
der Herkunft des Autors nicht weiter verwundert. Demgegenüber ist die empirische Ausbeute in den anderen vier Ländern insofern eher begrenzt als sich der Autor hier weitgehend auf vorhandene englischsprachige Literatur stützen muss. Nichtsdestoweniger sind die vergleichenden Länderanalysen ausgesprochen anregend und fördern ein vertieftes Verständnis dieser bisher wenig beackerten Reformlandschaft.

Das Buch ist bewusst auf den akademisch orientierten Leser ausgerichtet. Es geht weniger um umfassende Beschreibung von Reformelementen, sondern um eine theoriebasierte Analyse und Erklärung beobachteter Reformtendenzen sowie um die Herausarbeitung von Gemeinsamkeiten und Unterschieden der Managementreformen in den betrachteten Ländern. Das Buch ist vor allem für den wissenschaftlichen Diskurs über Public Management-Reformen in Europa wertvoll, aber auch für vertiefende Lehrveranstaltungen in Masterprogrammen, die sich mit Verwaltungsreformen aus international-vergleichender Sicht befassen.

Christoph Reichard

Björn Raupach und Katrin Stangenberg,

Doppik in der öffentlichen Verwaltung - Grundlagen, Verfahrensweisen, Einsatzgebiete, 2. Aufl., Gabler Verlag, Wiesbaden 2009, $228 S$.

In Deutschland gibt es derzeit kaum eine Kommune, die sich nicht intensiv mit der Einführung der Doppik bzw. der erweiterten Kameralistik auseinandersetzt. Derzeit laufen in vielen Kommunen daher die umfangreichsten Reorganisationsprozesse seit vielen Jahren unter den verschiedensten Konnotationen, wie NKF, NKH, NKR, NKHR, NKRS oder einfach „kommunale Doppik“. Unabhängig von den landesspezifischen Bezeichnungen soll mit der kommunalen Doppik eigentlich keine neue Bürokratie geschaffen, sondern ein Anstoß zur Professionalisierung der kommunalen Verwaltung gegeben werden - festgelegt in den Beschlüssen der Innenministerkonferenz vom 21.11.2003. Die Praxis zeigt allerdings, dass die Umstellung der bisher zahlungsorientierten Darstellungsform der herkömmlichen Kameralistik auf eine ressourcenorientierte Darstel- lung verbunden mit einer Steuerung der Kommunen durch die Vorgabe von Zielen für kommunale Leistungen (Output- bzw. Outcomesteuerung) einen erheblichen Kraftakt für die meisten Kommunen darstellt. Die Beschlüsse der Innenministerkonferenz sowie die Vorgaben der jeweiligen Landesprojekte geben nur wenig konkrete Hinweise, wie eine Kommune einer bestimmten Größenordnung die Doppikumstellung beschreiten sollte, um einen größtmöglichen Nutzen mit Blick auf eine verbesserte Verwaltungssteuerung erzielen zu können.

Björn Raupach und Katrin Stangenberg nehmen sich in dem vorliegenden Buch dieser Problemstellung an und setzen sich zum Ziel, am Prozess der Verwaltungsreform Interessierten einen Leitfaden darzureichen, der die Beschreitung des Reformweges erleichtern soll. Das Buch ist damit vorrangig als praxisorientierte Abhandlung intendiert und erhebt keinen Anspruch auf einen forschungsorientierten wissenschaftlichen Beitrag zur Reform des öffentlichen Haushalts- und Rechnungswesens. Mit Blick auf die Zielsetzung und die Zielgruppe liegt ein durchaus ansprechendes Werk vor, das angereichert durch Erfahrungen und Beispiele aus laufenden und abgeschlossenen Umstellungsprozessen sowie die in der Praxis zu beachtenden Rahmenbedingungen nicht aus dem Auge verlierend gezielte Empfehlungen für die Umstellung von der Kameralistik auf die Doppik gibt.

Das Buch ist in drei Teile untergliedert, einem die Thematik in die aktuellen Reformdiskussion einordnenden und Grundlagen des (neuen) öffentlichen Haushaltswesens darlegenden Überblick, einem die Grundlagen der Doppik darlegenden zweiten Teil und einem gezielte Handreichungen für die Doppik in der öffentlichen Verwaltung gebenden abschließenden dritten Teil. Herauszustellen für alle Teile sind die von den Autoren gezielt und stimmig positionierten Beispiele zu den angesprochenen Themen. Aufgrund der Zielrichtung des Buches und seines Anspruches, einen Leitfaden für Umstellungsprozesse darzureichen, stellt sich den Autoren allerdings die in keiner Weise zu unterschätzende Herausforderung, wohlüberlegt $\mathrm{zu}$ entscheiden, welche Themen in dem Buch angesprochen und in einem zweiten Schritt, in welcher Breite und Tiefe diese zu behandeln sind. So nehmen die gezielt für 
„Nicht-Buchhalter“ geschriebenen Kapitel zum Jahresabschluss, der Systematik der Buchführung, der Kosten- und Leistungsrechnung etc. freilich viel Raum ein. Für die Leserschaft wäre es hier ggf. hilfreich, in der Einführung Hinweise zu geben, welche Abschnitte des Buches für welche Zielgruppe, z.B. in Abhängigkeit des Grades an Rechnungswesen-Kenntnissen, relevant sind, um so eine gezielte Bearbeitung des Buches zu ermöglichen. Die Problematik der Abwägung von „Breite und Tiefe" wird insbesondere auch an dem Kapitel 3.3. verdeutlicht, in dem die Autoren über vier Seiten die International Public Sector Accounting Standards (IPSAS) thematisieren: Mit Blick auf die Praxisrelevanz für Kommunen sind diese aktuell nicht zuvorderst auf der Agenda im Umstellungsprozess platziert, mit Blick auf deren Kennzeichnung, der einordnenden Erläuterung zu Bewertung und Ansatz sind wiederum vier Seiten zu eng bemessen. Ggf. hätte eine die aktuelle Diskussion um die Zweckmäßigkeit der Anwendung von spezifisch für den öffentlichen Sektor entwickelten Standards, wie z.B. die IPSAS oder die GoöB, kurz und prägnant einordnende, zusammenfassende Ausführung mit Blick auf die Zielset- zung des Buches ausgereicht. Gleiches gilt für das nur drei Seiten umfassende Kapitel 11 zur Konzernbilanz, das der der dort behandelten Thematik nur schwerlich gerecht werden kann. Nicht als Kritik, sondern vielmehr als Anregung für Folgeauflagen sei an dieser Stelle die Empfehlung ausgesprochen, im Anschluss an die jeweiligen Kapitel gezielte Literaturhinweise, neben Büchern auch aktuelle Zeitschriftenartikel und Internetverweise, zu geben, die interessierten Lesern die Möglichkeit einer vertieften Auseinandersetzung mit der Thematik und deren spezifischen Fragestellungen und/oder eines weiteren Ausbaus der frisch erworbenen Grundlagenkenntnisse eröffnen. In der Gesamtschau kann festgehalten werden, dass das vorliegende Werk allen einschlägig Interessierten in der Praxis einen strukturierten, praktische Fragen des Umsetzungsprozesses explizit berücksichtigenden und mit anschaulichen Beispielen angereicherten Leitfaden zur Hand gibt, dessen Lektüre allen am Umsetzungsprozess von der Kameralistik auf die Doppik Beteiligten und davon Betroffenen zu empfehlen ist.

Christina Schaefer 